\title{
Adaptive servo ventilation for central sleep apnoea in heart failure: SERVE-HF on-treatment analysis
}

\author{
Holger Woehrle ${ }^{1,2}$, Martin R. Cowie ${ }^{3}$, Christine Eulenburg ${ }^{4}$, Anna Suling ${ }^{5}$, \\ Christiane Angermann ${ }^{6}$, Marie-Pia d'Ortho ${ }^{7}$, Erland Erdmann ${ }^{8}$, Patrick Levy ${ }^{9}$, \\ Anita K. Simonds ${ }^{10}$, Virend K. Somers ${ }^{11}$, Faiez Zannad ${ }^{12}$, Helmut Teschler ${ }^{13}$ \\ and Karl Wegscheider ${ }^{5}$
}

Affiliations: ${ }^{1}$ ResMed Science Center, ResMed Germany Inc., Martinsried, Germany. ${ }^{2}$ Sleep and Ventilation Center Blaubeuren, Respiratory Center Ulm, Ulm, Germany. ${ }^{3}$ Imperial College London, London, UK. ${ }^{4}$ Department of Epidemiology, University Medical Center Groningen, Groningen, the Netherlands. ${ }^{5}$ Department of Medical Biometry and Epidemiology, University Medical Center Eppendorf, Hamburg, Germany. ${ }^{6}$ Department of Medicine I and Comprehensive Heart Failure Center, University Hospital and University of Würzburg, Würzburg, Germany. ${ }^{7}$ University Paris Diderot, Sorbonne Paris Cité, Hôpital Bichat, Explorations Fonctionnelles, DHU FIRE, AP-HP, Paris, France. ${ }^{8}$ Heart Center, University of Cologne, Cologne, Germany. ${ }^{9} \mathrm{CHU}$ de Grenoble, Grenoble, France. ${ }^{10}$ Royal Brompton Hospital, London, UK. ${ }^{11}$ Mayo Clinic and Mayo Foundation, Rochester, MN, USA. ${ }^{12}$ INSERM, Université de Lorraine, CHU Nancy, France. ${ }^{13}$ Department of Pneumology, Ruhrlandklinik, West German Lung Center, University Hospital Essen, University Duisburg-Essen, Essen, Germany.

Correspondence: Holger Woehrle, Sleep and Ventilation Center Blaubeuren/Lung Center Ulm, Olgastr. 83, 89073 Ulm, Germany. E-mail: hwoehrlealungenzentrum-ulm.de

@ERSpublications

SERVE-HF on-treatment results showed increased cardiovascular risk similar to the intention-to-treat analysis http://ow.ly/hoO130 dx4s9

Cite this article as: Woehrle $\mathrm{H}$, Cowie MR, Eulenburg C, et al. Adaptive servo ventilation for central sleep apnoea in heart failure: SERVE-HF on-treatment analysis. Eur Respir J 2017; 50: 1601692 [https://doi.org/ $10.1183 / 13993003.01692-2016]$.

ABSTRACT This on-treatment analysis was conducted to facilitate understanding of mechanisms underlying the increased risk of all-cause and cardiovascular mortality in heart failure patients with reduced ejection fraction and predominant central sleep apnoea randomised to adaptive servo ventilation versus the control group in the SERVE-HF trial.

Time-dependent on-treatment analyses were conducted (unadjusted and adjusted for predictive covariates). A comprehensive, time-dependent model was developed to correct for asymmetric selection effects (to minimise bias).

The comprehensive model showed increased cardiovascular death hazard ratios during adaptive servo ventilation usage periods, slightly lower than those in the SERVE-HF intention-to-treat analysis. Self-selection bias was evident. Patients randomised to adaptive servo ventilation who crossed over to the control group were at higher risk of cardiovascular death than controls, while control patients with crossover to adaptive servo ventilation showed a trend towards lower risk of cardiovascular death than patients randomised to adaptive servo ventilation. Cardiovascular risk did not increase as nightly adaptive servo ventilation usage increased.

On-treatment analysis showed similar results to the SERVE-HF intention-to-treat analysis, with an increased risk of cardiovascular death in heart failure with reduced ejection fraction patients with predominant central sleep apnoea treated with adaptive servo ventilation. Bias is inevitable and needs to be taken into account in any kind of on-treatment analysis in positive airway pressure studies. 


\section{Introduction}

Central sleep apnoea (CSA) has been identified as an independent predictor of increased morbidity and mortality in patients with heart failure [1-5], and there are a number of plausible mechanisms by which CSA could have detrimental effects on cardiac function $[1,6,7]$. Therefore, the effect of interventions to treat CSA on outcomes has been investigated in heart failure patients and, until recently, an accumulating body of evidence has suggested that effective treatment of CSA and/or Cheyne-Stokes respiration (CSR) with positive airway pressure therapy is associated with beneficial effects in this patient population [8-17].

Adaptive servo ventilation (ASV) has been shown to be the most effective form of positive airway pressure therapy for alleviating CSA/CSR in heart failure [18-20]. However, the main results of the recent Treatment of Sleep-Disordered Breathing With Predominant Central Sleep Apnea by Adaptive Servo Ventilation in Patients With Heart Failure (SERVE-HF; NCT00733343) study raised questions about the value of this treatment approach in patients with heart failure with reduced ejection fraction (HFrEF) and predominant CSA [21]. SERVE-HF was the first large, randomised clinical trial of ASV therapy that included survival and cardiovascular outcomes. The study result for the primary endpoint (a composite of all-cause mortality, life-saving cardiovascular intervention or unplanned hospitalisation for worsening chronic heart failure) was neutral, with no statistically significant differences between the ASV and control groups (HR 1.13, 95\% CI 0.97, 1.32; p=0.113) [21]. However, although there were no adverse events associated with the performance of the ASV device, SERVE-HF unexpectedly showed an increase in mortality in ASV recipients. Both all-cause mortality $(1.28$; 95\% CI 1.06-1.55; p=0.01) and cardiovascular mortality (HR 1.34, 95\% CI 1.09-1.65; p=0.006) were significantly higher in the ASV than in the control group [21]. Because the main SERVE-HF study was designed to compare outcomes in the ASV and control groups, all analyses were conducted on an intention-to-treat (ITT) basis. The results of this analysis clearly showed that patients allocated to the ASV group had worse outcomes than those allocated to control group, but it did not provide any information on the mechanisms underlying the increase in cardiovascular mortality, or on the impact of device usage hours on outcomes. To try and answer these questions we can take advantage of the substantial crossover between treatment groups that occurred in the study. In trials where problems with compliance or adherence with the study interventions are to be expected, additional on-treatment (OT) analyses are frequently performed to distinguish between effects caused directly by the assigned treatment and indirect effects of changes in activities of daily life, general fitness or adherence to medication, or selected or restricted use of treatment [22]. This distinction between direct and indirect effects may have important consequences for future users. However, it is sometimes forgotten that on-treatment analyses (which essentially are comparisons of users and non-users, or of periods with and without device use) are not protected by randomisation, but are instead governed by patient and physician preferences. Thus, these analyses should be evaluated similarly to cohort studies, with adequate adjustment and a discussion of biases that might have influenced the results $[22,23]$.

A series of analyses of the primary composite endpoint of SERVE-HF data was prespecified in the study protocol. After the results of the primary analysis became available [21] the focus has shifted to cardiovascular mortality. The analyses presented in this paper used different definitions of "on-treatment" to investigate potential effects of actual treatment received during the study on cardiovascular mortality and effects due to selective use of the ASV device.

\section{Materials and methods}

\section{Study design and participants}

SERVE-HF was a multinational, multicentre, randomised, parallel-group, event-driven study. Full details of the study design have been reported previously $[21,24]$. The SERVE-HF study protocol was approved by the ethics committee at each participating centre. The trial was conducted according to Good Clinical Practice and the Principles of the Declaration of Helsinki. All participants gave written informed consent.

\section{Randomisation and masking}

Patients were randomised in a 1:1 ratio to the ASV or control group. Randomisation was performed using codes generated by a central computer. The study had an open-label design because of the practical, scientific and ethical issues, and problems with investigator blinding, associated with delivery of sham positive airway pressure therapy [25].

Received: Aug 242016 | Accepted after revision: May 282017

Support statement: The SERVE-HF study was supported by ResMed Ltd. Funding information for this article has been deposited with the Crossref Funder Registry.

Conflict of interest: Disclosures can be found alongside this article at erj.ersjournals.com 


\section{Procedures}

SERVE-HF participants were randomised to receive optimal medical therapy for heart failure alone, or in combination with ASV (Auto Set CS, ResMed). For full details of ASV titration and settings, please see the primary study publication [21]. ASV usage information was downloaded from the device at each follow-up.

\section{Outcomes}

Cardiovascular death was a secondary endpoint in the SERVE-HF study. Death was classified as cardiovascular unless an unequivocal non-cardiovascular cause of death was confirmed by the central adjudication committee. Cardiovascular death included sudden death; death because of myocardial infarction, heart failure or stroke; procedure-related death (death during a cardiovascular investigation/ procedure/operation); death because of other specified cardiovascular causes; and presumed cardiovascular deaths (e.g. those for which a non-cardiovascular cause could not be clearly established).

\section{Statistical analysis}

Unadjusted and adjusted Cox cause-specific hazards models were applied to different subsets to evaluate treatment effects. Firstly, an intention-to-treat (ITT) analysis, consisting of all randomised patients, was performed (figure 1b, bottom). For the OT analyses, the follow-up data set was divided into intervals of continuous device usage, allowing ASV usage to be introduced as a time-dependent variable (figure 1a). The as-treated analysis compared all treatment periods with ASV use with all treatment periods without ASV use, regardless of which group the patient had been randomised to (figure $1 \mathrm{~b}$, middle). This analysis does not take randomisation as the dominant condition into account and thus is highly vulnerable to adherence bias. To investigate this bias, we compared hazard rates for patients who were at least initially adherent to the randomised therapy with those who crossed over to the same therapy right from the beginning ("early crossovers", see below). If there was no selection bias, there should be no substantial outcome differences between these groups.

The as-treated-as-randomised comparison was similar, but also took the randomisation group into account. Therefore, it was based on actual treatment received for each time period (as above) but only included intervals where patients used the treatment to which they had been initially randomised (figure $1 \mathrm{~b}$, top). Since this comparison does not include all patients with all measurements, we extended this model to a comprehensive regression model that included all time periods. This was possible because the model was extended by additional time-dependent covariates that mark early and late crossover periods for each group separately. It can be expected that all OT comparisons are influenced by the decision of a patient or physician to use or not to use the ASV device, which will be associated with a variety of characteristics and factors and that, in turn, may be related to outcomes. Therefore, a series of predictive baseline variables was added to the comprehensive model for adjustment. Of the list of considered baseline variables, the only ones omitted were those that did not significantly contribute to the prediction model and did not change the effect estimate of the intervention by more than $10 \%$. To better understand reasons for non-adherence, we analysed patients who had early versus late crossover to the other treatment group separately. Early crossover was defined as a documented change of allocated treatment within the first 2 weeks after randomisation and considered as a refusal of the randomised therapy without any attempt to use it ("never tried"), and late crossover was defined as a documented change of allocated treatment occurring more than 2 weeks after randomisation (considered to have occurred in reaction to experience with the randomised treatment) (figure 1a). Finally, time-varying data on average daily device usage in the ASV group were used to investigate dose effects of ASV usage on the outcome. For this purpose, the comprehensive model was extended by further differentiation of ASV use.

All analyses were performed both unadjusted and adjusted for the same selection of variables. In multivariable models, variables adjusted for were age (years), sex (female/male), body mass index (BMI; $\mathrm{kg} \cdot \mathrm{m}^{-2}$ ), diabetes (yes/no), NYHA class $(\geqslant 3 /<3$ ), left ventricular ejection fraction (LVEF), central AHI as a percentage of the total AHI, glomerular filtration rate (GFR), time spent with oxygen saturation of $<90 \%$ (min), bundle branch block (none, right, left, other), ischaemia (yes/no). Continuous variables were centred around the mean value in the control group. Missing values were replaced by means. A missing indicator was generated for each continuous covariable with missing values. Missing values for categorical variables were summarised in one category.

\section{Results}

The ITT, as-treated and overall as-treated-as-randomised (comprehensive OT) analyses included 1676 observed intervals of constant use in 1325 subjects. A total of 583 patients randomised to ASV completed the study, $168(28.8 \%)$ of whom discontinued device use during the study; of the 578 patients randomised to control who completed the study, $98(17.0 \%)$ started ASV $(n=87)$, bilevel spontaneous timed (ST) $(n=2)$ 

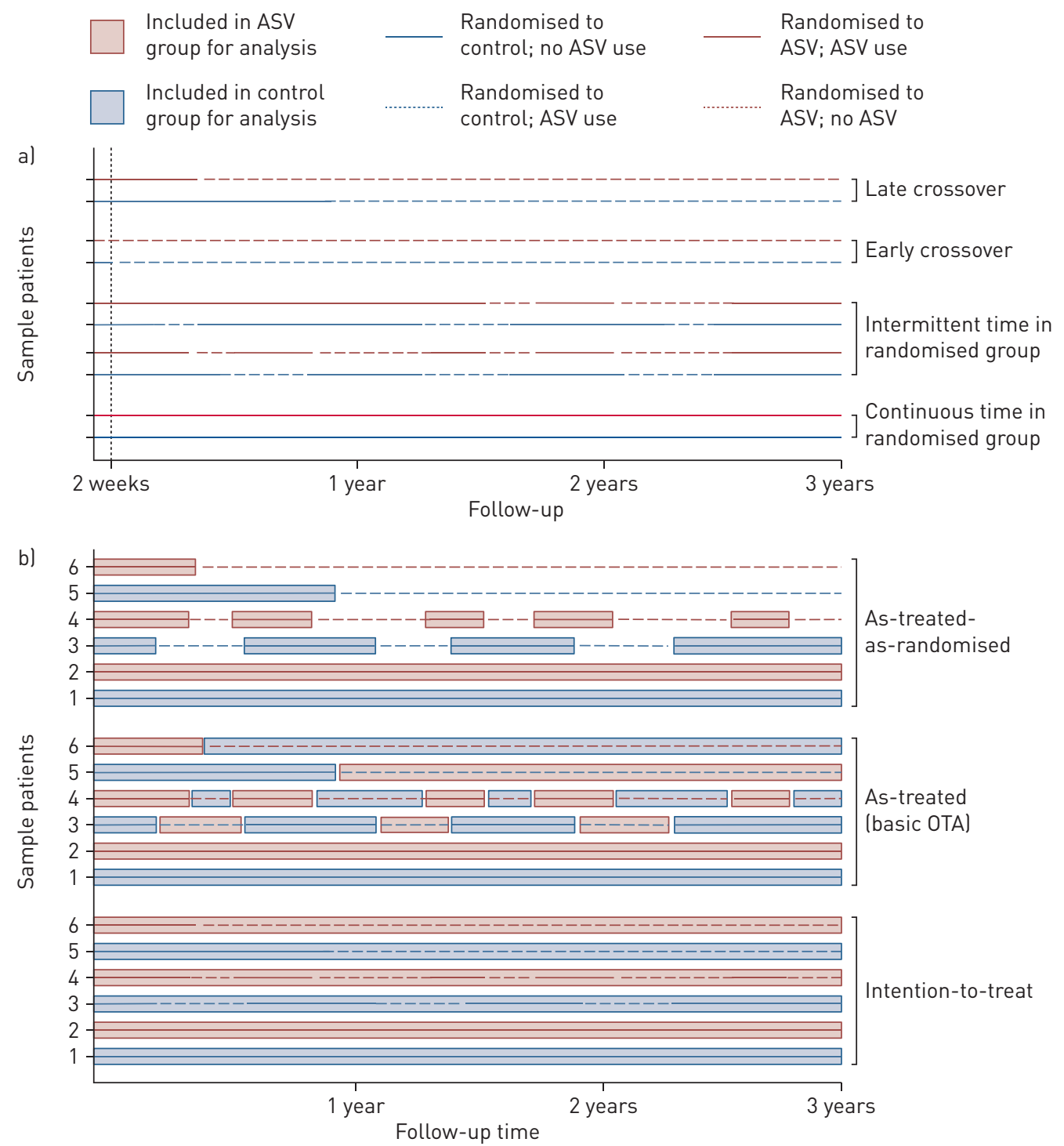

FIGURE 1 Schematic representing different possible patterns of device usage and intervals in the different on-treatment analyses (OTA) (follow-up times are indicative only). Solid lines show periods where treatment was as randomised (control, blue; or ASV, red) and dotted lines show periods were treatment was not as randomised (e.g. no ASV use in the ASV group, or ASV use in the control group). a) Possible usage and adherence patterns to allocated treatment. Patients with continuous use remained in their randomised group and adherent to treatment throughout the study. The intermittent use examples show patients in both the ASV (red line) and control groups (blue line) switching between their allocated treatment (solid lines) and the alternative treatment (dotted lines). Early crossover in the ASV group refers to patient refusal of ASV despite randomisation to the ASV group, and early crossover in the control group refers to initiation of ASV within the first 2 weeks after randomisation in a patient randomised to the control group. Late crossover in the ASV group refers to withdrawal of ASV $>2$ weeks after randomisation in patients randomised to the ASV group, and late crossover in the control group refers to initiation of ASV $>2$ weeks after randomisation in patients randomised to the control group. b) Treatment intervals for six different hypothetical patients included in the on-treatment analyses (intention-to-treat, as-treated, as-treated-as-randomised). Intention-to-treat analysis allocates all follow-up intervals to the randomised treatment group, regardless of usage. As-treated analysis includes periods allocated on the basis of actual treatment during different follow-up intervals (ASV, red boxes; or control, blue boxes). As-treated-as-randomised analysis also allocates intervals on the basis of actual treatment received but only includes intervals where patients used the treatment to which they had been initially randomised (ASV, red boxes; or control, blue boxes). For all examples, patients 1 and 2 remained continuously in their randomised group, patients 3 and 4 had intermittent time in their randomised group and patients 5 and 6 crossed over from randomised treatment to the alternative group.

or continuous positive airway pressure $(n=8)$ therapy during the study (all of which was considered ASV use for the analyses; type of positive airway pressure therapy was unknown in one patient).

There were a large number of patient factors identified as having a significant influence on cardiovascular death risk (table 1). The ITT analysis (both unadjusted and adjusted) showed a significantly increased risk 


\begin{tabular}{|c|c|c|}
\hline & Hazard ratio $(95 \% \mathrm{CI})$ & p-value \\
\hline Age, per 5-year increase & $1.08(1.02-1.15)$ & 0.013 \\
\hline Female versus male & $0.52(0.34-0.82)$ & 0.005 \\
\hline BMI, per $5 \mathrm{~kg} \cdot \mathrm{m}^{-2}$ increase & $0.80(0.69-0.92)$ & 0.001 \\
\hline NYHA class $\geqslant 3$ versus 2 & $1.43(1.11-1.85)$ & 0.005 \\
\hline LVEF, per $5 \%$ increase & $0.86(0.79-0.93)$ & $<0.001$ \\
\hline Ischaemic versus non-ischaemic HF aetiology & $1.41(1.12-1.78)$ & 0.003 \\
\hline \multicolumn{3}{|l|}{ Bundle-branch block, versus none } \\
\hline Right & $1.57(1.05-2.35)$ & 0.029 \\
\hline Left & $1.51(1.15-1.98)$ & 0.003 \\
\hline Other & $1.50(1.13-1.98)$ & 0.004 \\
\hline Diabetes, yes versus no & $1.32(1.06-1.64)$ & 0.012 \\
\hline GFR, per $1 \mathrm{~mL} / \mathrm{min} / 1.73 \mathrm{~m}^{2}$ increase & $0.98(0.97-0.99)$ & $<0.001$ \\
\hline Central $\mathrm{AHI} /$ total $\mathrm{AHI}$, per $10 \%$ increase & $1.01(1.00-1.02)$ & 0.011 \\
\hline Time spent with oxygen saturation $<90 \%$, per $\min$ increase & $1.003(1.001-1.004)$ & $<0.001$ \\
\hline
\end{tabular}

AHI: apnoea-hypopnoea index; BMI: body mass index; GFR: glomerular filtration rate; LVEF: left ventricular ejection fraction; HF: heart failure; NYHA: New York Heart Association.

of cardiovascular death in the ASV group (table 2). In the as-treated analysis (based on actual treatment received during all follow-up intervals, irrespective of randomisation), the increased cardiovascular risk in the ASV group was no longer evident (table 2). The main reason for this difference in results compared with the primary analysis was selection effects, as can be seen in table 3: treatment periods without ASV use in the control group are associated with much lower cardiovascular risk than treatment periods without ASV use in the ASV group. Given that both these patient groups received no study intervention, the most likely explanation for the observed effects is strong self-selection of crossover patients who refuse to start ASV. This results in "bad risks" being moved from the ASV random group to the control group of the as-treated analysis, favouring the ASV group of the as-treated analysis before any treatment was given. Similarly, cardiovascular risk was lower in control group patients who crossed over to ASV than in patients randomised to ASV who used the device, again highlighting the presence of selection and self-selection effects that influence the results. This time, "good risks" are moved from the control random group to the ASV group of the as-treated analysis, again favouring the ASV group of the as-treated analysis. The selection bias therefore reduces the mortality difference that was seen between random groups in the ITT analysis. To mitigate selection bias, the random groups must be taken into account, even in the OT analysis. The as-treated-as-randomised analysis, which compared only time intervals where patients used the treatment to which they had been initially randomised, also showed elevated hazard ratio (HR) values for ASV versus control but these were slightly lower than those in the ITT analysis (table 2).

TABLE 2 Risk of cardiovascular death based on randomised treatment groups

\begin{tabular}{|c|c|c|c|c|}
\hline & \multicolumn{4}{|c|}{ ASV versus control } \\
\hline & \multicolumn{2}{|c|}{ Unadjusted } & \multicolumn{2}{|c|}{ Adjusted } \\
\hline & HR $(95 \% \mathrm{CI})$ & p-value & HR $(95 \% \mathrm{CI})$ & p-value \\
\hline Intention-to-treat ${ }^{\#}$ & $1.34(1.09-1.65)$ & 0.006 & $1.37(1.10-1.69)$ & 0.004 \\
\hline As-treated ? & $0.96(0.78-1.19)$ & 0.738 & $1.09(0.88-1.35)$ & 0.440 \\
\hline As-treated-as-randomised ${ }^{+}$ & $1.17(0.92-1.48)$ & 0.198 & $1.27(1.00-1.62)$ & 0.051 \\
\hline Comprehensive model & $1.17(0.92-1.47)$ & 0.198 & $1.28(1.01-1.63)$ & 0.043 \\
\hline
\end{tabular}

ASV: adaptive servo ventilation; $\mathrm{Cl}$ : confidence interval; $\mathrm{HR}$ : hazard ratio. \#: Patients remained in the treatment group to which they were randomised, regardless of device usage or not. ": Analysis based on the treatment patients actually received during different follow-up intervals (ASV or control for each time interval), including all follow-up time intervals. ${ }^{+}$: Analysis based on the treatment patients actually received during different follow-up intervals (ASV or control for each time interval), only including time intervals where patients used the treatment to which they had been initially randomised. 
TABLE 3 Cardiovascular death incidence rates in usage and non-usage intervals of patients in different randomisation groups

Persons Person-years Cardiovascular Incidence rate per 100 death events person-years $(95 \% \mathrm{CI})$

\begin{tabular}{lcccc}
\hline $\begin{array}{l}\text { Patients starting without ASV use } \\
\quad \text { Randomised to control }\end{array}$ & 637 & 2002.47 & 156 & 7.79 (6.66-9.11) \\
$\quad \begin{array}{l}\text { Randomised to ASV ("early } \\
\quad \text { crossover" to control) }\end{array}$ & 53 & 143.22 & 14 & 9.78 (5.79-16.51) \\
$\begin{array}{l}\text { Patients starting with ASV use } \\
\quad \text { Randomised to control } \\
\quad \text { ("early crossover" to ASV) }\end{array}$ & 22 & 65.68 & 2 & 3.05 (0.76-12.18) \\
$\quad$ Randomised to ASV & 613 & 1814.30 & 185 & 10.20 (8.83-11.78)
\end{tabular}

ASV: adaptive servo ventilation; $\mathrm{Cl}$ : confidence interval.

Table 4 shows the results of the comprehensive model. Patients randomised to ASV who crossed over to control were at higher risk of cardiovascular death than control as-randomised, and even than ASV use as-randomised (particularly when crossover occurred within 2 weeks of randomisation) whereas the opposite was the case for control patients who crossed over to ASV, who showed a trend towards a reduced risk of cardiovascular death (table 4). Patients who did not use their randomised treatment showed the greatest divergence in event rates between the ASV and control groups, much larger than for patients who crossed over later, indicating that the bias results from self-selection and not from device-related adverse events.

In patients randomised to ASV $(n=666)$, interval-wise measured mean device usage was compared with device usage for $<3 \mathrm{~h} /$ night in patients randomised to ASV, and also compared with controls without ASV use in the total study population. No dose-dependency for the effect of ASV on cardiovascular risk was observed in either the adjusted or unadjusted analyses (table 5).

\section{Discussion}

The OT analysis of the SERVE-HF trial shows that the increase in cardiovascular mortality seen in patients randomised to ASV in the ITT analysis mainly occurs during periods of device usage, suggesting a causal relationship between ASV use and cardiovascular death, although the risk did not appear to be proportional to the nightly duration of device usage.

\begin{tabular}{|c|c|c|}
\hline & $\begin{array}{l}\text { Adjusted HR } \\
\text { (95\% CI) }\end{array}$ & p-value \\
\hline ASV versus control (both as-randomised) & $1.28(1.01-1.63)$ & 0.043 \\
\hline \multicolumn{3}{|l|}{ Crossover of ASV patients } \\
\hline ASV withdrawal versus control as-randomised & $1.44(1.06-1.94)$ & 0.019 \\
\hline $\begin{array}{l}\text { Early crossover" from ASV to control ("never tried ASV") versus control } \\
\text { as-randomised }\end{array}$ & $1.67(0.95-2.91)$ & 0.073 \\
\hline $\begin{array}{l}\text { Late crossover from ASV to control ("discontinued ASV") versus control } \\
\text { as-randomised }\end{array}$ & $1.38(0.99-1.92)$ & 0.056 \\
\hline \multicolumn{3}{|l|}{ Crossover of control patients } \\
\hline Crossover from control to ASV versus control as-randomised & $0.74(0.42-1.30)$ & 0.295 \\
\hline Early crossover ${ }^{\#}$ from control to ASV versus control as-randomised & $0.44(0.11-1.83)$ & 0.258 \\
\hline Late crossover ${ }^{\text {Il }}$ from control to ASV versus control as-randomised & $0.83(0.46-1.52)$ & 0.551 \\
\hline
\end{tabular}

ASV: adaptive servo ventilation; $\mathrm{Cl}$ : confidence interval; HR: hazard ratio. ${ }^{\#}$ : Early crossover in the ASV group refers to patient refusal of ASV despite randomisation to the ASV group, and early crossover in the control group refers to initiation of ASV within the first 2 weeks after randomisation in a patient randomised to control. ": Late crossover in the ASV group refers to withdrawal of ASV $>2$ weeks after randomisation in patients randomised to the ASV group, and late crossover in the control group refers to initiation of $A S V>2$ weeks after randomisation in patients randomised to control. 
TABLE 5 Risk of cardiovascular death based on device usage intervals

\begin{tabular}{|c|c|c|c|c|}
\hline & \multicolumn{2}{|c|}{ Unadjusted } & \multicolumn{2}{|c|}{ Adjusted } \\
\hline & $\operatorname{HR}(95 \% \mathrm{CI})$ & p-value & $\operatorname{HR}(95 \% \mathrm{CI})$ & p-value \\
\hline \multicolumn{5}{|c|}{ ASV as-randomised by ASV usage } \\
\hline \multicolumn{5}{|c|}{ ASV usage interval (average daily usage; h/night) } \\
\hline$>0$ to $<3$ ( $23 \%$ of observation time) & 1 (reference) & & 1 (reference) & \\
\hline 3 to $<6$ ( $29 \%$ of observation time) & $1.07(0.69-1.67)$ & 0.766 & $1.06(0.68-1.67)$ & 0.789 \\
\hline$\geqslant 6(28 \%$ of observation time $)$ & $1.15(0.74-1.78)$ & 0.540 & $1.03(0.66-1.61)$ & 0.898 \\
\hline \multicolumn{5}{|c|}{ ASV as-randomised by ASV usage versus control as-randomised (all study patients) } \\
\hline$>0$ to $<3(23 \%$ of observation time $)$ & $1.08(0.74-1.57)$ & 0.686 & $1.23(0.84-1.81)$ & 0.282 \\
\hline 3 to $<6$ ( $29 \%$ of observation time) & $1.16(0.84-1.61)$ & 0.361 & $1.33(0.95-1.85)$ & 0.095 \\
\hline$\geqslant 6(28 \%$ of observation time $)$ & $1.25(0.91-1.71)$ & 0.170 & $1.28(0.93-1.77)$ & 0.125 \\
\hline
\end{tabular}

ASV: adaptive servo ventilation; $\mathrm{Cl}$ : confidence interval; HR: hazard ratio.

OT analyses of trial data initially analysed on an ITT basis are usually performed to distinguish between the direct effects of a treatment and indirect effects occurring during periods of non-adherence or treatment interruptions, which are frequently seen as avoidable. However, because the decision to stop a treatment is not independent of personal preferences and past experiences of physicians and patients, the comparison of periods with and without use of a treatment may be biased. This bias may even be stronger than the direct treatment effect. OT analyses are thus generally considered to provide less robust evidence than ITT analyses, similar to cohort studies. To attenuate or even eliminate the bias in OT analyses, epidemiological methods developed for the analysis of prospective observational studies can, and should, be applied, such as adjustment for baseline covariates or explicit modelling of selection effects.

This was the approach taken for OT analyses of SERVE-HF data. The "as-treated" analysis is the naïve analysis that does not take randomisation into account. Interpreting the results as causal effects ignores the fact that the risk of cardiovascular death associated with no device use in patients randomised to ASV was substantially higher than that in those randomised to the control group (table 3). This cannot be explained as a withdrawal effect because patients randomised to ASV who never started therapy were at higher risk of cardiovascular death than those with later crossover (table 4). Similarly, patients randomised to control who switched to ASV had lower cardiovascular mortality than those initially randomised to ASV. This suggests the presence of (self)-selection effects. In both groups, patients at lower risk (i.e. better disease state) preferred to use ASV, while patients at higher risk (i.e. worse disease state) preferred not to use ASV. Therefore, higher risks are shifted from ASV use intervals to non-use intervals just by the decision to refuse or interrupt ASV treatment. This significant bias resulting from preferential use is even stronger than the treatment effect and thus is able to overwhelm the ASV effect in the naïve unadjusted as-treated analysis. To overcome crossover bias, the as-treated-as-randomised analysis restricted to periods of randomised treatment was performed, embedded in a comprehensive model that included all observed periods and was adjusted for baseline covariates.

Crossover patients randomised to ASV who never started, or stopped, therapy did poorly in terms of cardiovascular risk. Conversely, crossovers from the control group who used the device appeared to do comparatively well in terms of cardiovascular risk. Such patients randomised to the control group who went on to use ASV are perhaps particularly motivated to improve their health by a variety of means and have significantly improved survival, whereas those in the ASV group who never use ASV therapy may care less about their health (or are overwhelmed by their medical problems) and therefore have a worse prognosis, confounding any treatment-related effects. These possibilities highlight the fact that, without the protection provided by randomisation, there are a wide variety of factors that might influence adherence and outcomes in patients using ASV therapy other than just device usage that have the potential to impact on the results of OT analyses. In addition, selection bias could be one of the things contributing to the fact that observational data were more favourable for ASV than data from the SERVE-HF randomised clinical trial.

Some of the factors that might influence adherence and outcomes, such as age [26], gender [26] and baseline sleep-disordered breathing severity [27], can be adjusted for in statistical models, whereas others, such as psychological variables [28], are more difficult to objectively quantify and account for. Likewise, changes in patient condition over a trial follow-up period may not be predicted by baseline characteristics. Device usage may not only be a marker of PAP therapy adherence, but also of a wide range of changes in 
comorbidities and of attitudes towards health and disease, and other less quantifiable factors. For example, patients with resistant hypertension randomised to sham renal denervation in the SIMPLICITY HTN-3 study showed improvements in medication adherence and optimisation of antihypertensive drug therapy during the trial such that reductions in blood pressure were not significantly different from those in the intervention group [29].

An important finding of this OT analysis of SERVE-HF is that there was no clear dose-response effect for ASV use and cardiovascular mortality. That is, patients who used ASV for longer each night were not more likely to experience cardiovascular death than those with lower usage levels. This is in contrast to findings from trials of PAP in patients with obstructive sleep apnoea where a dose-response effect for PAP has been reported, both for cardiovascular responses [30] and symptoms [31]; this was not seen for the negative effects of ASV on mortality in this analysis. However, the lack of dose dependency may have been a result of the way usage times were measured. Built-in device counts were used in the analysis and provided a summary of usage for the time between visits, rather than specific usage on each day. In addition, devices from some late patients were not available for final read-out. Therefore, we were unable to determine exact ASV device usage on the day of death, meaning we could have potentially missed any dose dependence of effect.

Adherence to ASV during SERVE-HF was $\geqslant 3$ hours/night in $60 \%$ of patients and was consistent over time [21]. Although not high, average ASV usage of 3.9 and 3.4 hours/night at 3 and 12 months, respectively, in SERVE-HF was similar to, or higher than, that in other randomised trials of PAP in older patients. PAP usage rates in the SAVE study, which included patients with cardiovascular disease, were 3.8 and 3.3 hours/night at 6 and 12 months [32], and in the PREDICT trial, elderly patients with obstructive sleep apnoea (mean age 71 years) were using continuous positive airway pressure for 1.5 hours/night at both 3 and 12 months [33].

Although an OT analysis was required to better understand the unexpected increase in death seen in SERVE-HF, the findings highlight a number of limitations and challenges with this approach. These include lack of protection provided by randomisation, differential effects of selection and self-selection, the possibility of reverse causation (e.g. if patients stop ASV use because they need intensive care), and limited sample sizes in crossover populations. Overall, OT analyses cannot replace, but only complement, the ITT analysis, which summarises direct and indirect effects resulting from the decision to start ASV treatment in a heart failure patient fulfilling the inclusion criteria. In addition to these bias-related issues, other limitations of this OT analysis include its post-hoc nature and the small sample size of patient subgroups in some analyses.

In conclusion, this OT analysis of SERVE-HF showed that actual ASV use is associated with increased cardiovascular risk, but did not demonstrate dose dependency of this effect.

\section{Acknowledgements}

The authors would like to thank the team from CRI (the clinical research institute) for their expertise in overseeing the SERVE-HF trial. Medical writing support was provided by Nicola Ryan, independent medical writer, funded by ResMed.

\section{References}

1 Bitter T, Westerheide N, Prinz C, et al. Cheyne-Stokes respiration and obstructive sleep apnoea are independent risk factors for malignant ventricular arrhythmias requiring appropriate cardioverter-defibrillator therapies in patients with congestive heart failure. Eur Heart J 2011; 32: 61-74.

2 Javaheri S, Shukla R, Zeigler H, et al. Central sleep apnea, right ventricular dysfunction, and low diastolic blood pressure are predictors of mortality in systolic heart failure. J Am Coll Cardiol 2007; 49: 2028-2034.

3 Yumino D, Wang H, Floras JS, et al. Relationship between sleep apnoea and mortality in patients with ischaemic heart failure. Heart 2009; 95: 819-824.

4 Hanly PJ, Zuberi-Khokhar NS. Increased mortality associated with Cheyne-Stokes respiration in patients with congestive heart failure. Am J Respir Crit Care Med 1996; 153: 272-276.

5 Lanfranchi PA, Braghiroli A, Bosimini E, et al. Prognostic value of nocturnal Cheyne-Stokes respiration in chronic heart failure. Circulation 1999; 99: 1435-1440.

6 Kasai T, Floras JS, Bradley TD. Sleep apnea and cardiovascular disease: a bidirectional relationship. Circulation 2012; 126: 1495-1510.

7 Somers VK, White DP, Amin R, et al. Sleep apnea and cardiovascular disease: an American Heart Association/ American College of Cardiology Foundation Scientific Statement from the American Heart Association Council for High Blood Pressure Research Professional Education Committee, Council on Clinical Cardiology, Stroke Council, and Council on Cardiovascular Nursing. J Am Coll Cardiol 2008; 52: 686-717.

8 Arzt M, Schroll S, Series F, et al. Auto-servoventilation in heart failure with sleep apnoea: a randomised controlled trial. Eur Respir J 2013; 42: 1244-1254.

9 Birner C, Series F, Lewis K, et al. Effects of auto-servo ventilation on patients with sleep-disordered breathing, stable systolic heart failure and concomitant diastolic dysfunction: subanalysis of a randomized controlled trial. Respiration 2014; 87: 54-62. 
10 Hastings PC, Vazir A, Meadows GE, et al. Adaptive servo-ventilation in heart failure patients with sleep apnea: a real world study. Int J Cardiol 2010; 139: 17-24.

11 Kourouklis SP, Vagiakis E, Paraskevaidis IA, et al. Effective sleep apnoea treatment improves cardiac function in patients with chronic heart failure. Int J Cardiol 2013; 168: 157-162.

12 Koyama T, Watanabe H, Igarashi G, et al. Short-term prognosis of adaptive servo-ventilation therapy in patients with heart failure. Circ J 2011; 75: 710-712.

13 Oldenburg O, Schmidt A, Lamp B, et al. Adaptive servoventilation improves cardiac function in patients with chronic heart failure and Cheyne-Stokes respiration. Eur J Heart Fail 2008; 10: 581-586.

14 Pepperell JC, Maskell NA, Jones DR, et al. A randomized controlled trial of adaptive ventilation for Cheyne-Stokes breathing in heart failure. Am J Respir Crit Care Med 2003; 168: 1109-1114.

15 Takama N, Kurabayashi M. Effectiveness of adaptive servo-ventilation for treating heart failure regardless of the severity of sleep-disordered breathing. Circ J 2011; 75: 1164-1169.

16 Topfer V, El-Sebai M, Wessendorf TE, et al. Adaptive servoventilation: effect on Cheyne-Stokes-respiration and on quality of life. Pneumologie 2004; 58: 28-32.

17 Zhang XL, Yin KS, Li XL, et al. Efficacy of adaptive servoventilation in patients with congestive heart failure and Cheyne-Stokes respiration. Chin Med J (Engl) 2006; 119: 622-627.

18 D'Elia E, Vanoli E, La Rovere MT, et al. Adaptive servo ventilation reduces central sleep apnea in chronic heart failure patients: beneficial effects on autonomic modulation of heart rate. J Cardiovasc Med (Hagerstown) 2013; 14: 296-300

19 Fietze I, Blau A, Glos M, et al. Bi-level positive pressure ventilation and adaptive servo ventilation in patients with heart failure and Cheyne-Stokes respiration. Sleep Med 2008; 9: 652-659.

20 Teschler H, Dohring J, Wang YM, et al. Adaptive pressure support servo-ventilation: a novel treatment for Cheyne-Stokes respiration in heart failure. Am J Respir Crit Care Med 2001; 164: 614-619.

21 Cowie MR, Woehrle H, Wegscheider K, et al. Adaptive servo-ventilation for central sleep apnea in systolic heart failure. N Engl J Med 2015; 373: 1095-1105.

22 Peduzzi P, Wittes J, Detre K, et al. Analysis as-randomized and the problem of non-adherence: an example from the Veterans Affairs Randomized Trial of Coronary Artery Bypass Surgery. Stat Med 1993; 12: 1185-1195.

23 Porta N, Bonet C, Cobo E. Discordance between reported intention-to-treat and per protocol analyses. J Clin Epidemiol 2007; 60: 663-669.

24 Cowie MR, Woehrle H, Wegscheider K, et al. Rationale and design of the SERVE-HF study: treatment of sleep-disordered breathing with predominant central sleep apnoea with adaptive servo-ventilation in patients with chronic heart failure. Eur J Heart Fail 2013; 15: 937-943.

25 Djavadkhani Y, Marshall NS, D'Rozario AL, et al. Ethics, consent and blinding: lessons from a placebo/sham controlled CPAP crossover trial. Thorax 2015; 70: 265-269.

26 Woehrle H, Graml A, Weinreich G. Age- and gender-dependent adherence with continuous positive airway pressure therapy. Sleep Med 2011; 12: 1034-1036.

27 Schoch OD, Baty F, Niedermann J, et al. Baseline predictors of adherence to positive airway pressure therapy for sleep apnea: a 10-year single-center observational cohort study. Respiration 2014; 87: 121-128.

28 Poulet C, Veale D, Arnol N, et al. Psychological variables as predictors of adherence to treatment by continuous positive airway pressure. Sleep Med 2009; 10: 993-999.

29 Bhatt DL, Kandzari DE, O'Neill WW, et al. A controlled trial of renal denervation for resistant hypertension. N Engl J Med 2014; 370: 1393-1401.

30 Middleton S, Vermeulen W, Byth K, et al. Treatment of obstructive sleep apnoea in Samoa progressively reduces daytime blood pressure over 6 months. Respirology 2009; 14: 404-410.

31 Weaver TE, Maislin G, Dinges DF, et al. Relationship between hours of CPAP use and achieving normal levels of sleepiness and daily functioning. Sleep 2007; 30: 711-719.

32 Chai-Coetzer CL, Luo YM, Antic NA, et al. Predictors of long-term adherence to continuous positive airway pressure therapy in patients with obstructive sleep apnea and cardiovascular disease in the SAVE study. Sleep 2013; 36: 1929-1937.

33 McMillan A, Bratton DJ, Faria R, et al. Continuous positive airway pressure in older people with obstructive sleep apnoea syndrome (PREDICT): a 12-month, multicentre, randomised trial. Lancet Respir Med 2014; 2: 804-812. 